\title{
Automatic image segmentation system through iterative edge-region co-operation
}

\author{
Chafik Djalal Kermad*, Kacem Chehdi \\ LASTI-Groupe Image, ENSSAT, 6 rue de Kerampont, 22305 Lannion, France \\ Received 1 September 1999; received in revised form 4 March 2002; accepted 26 March 2002
}

\begin{abstract}
In this paper, we propose an image segmentation system adapted to the uniform and/or weakly textured region extraction.

The architecture of the proposed system combines two concepts. (i) The integration of the information resulting from two complementary segmentation methods: edge detection and region extraction. Thus, this allows us to exploit the advantages of each. (ii) The active perception via the intermediate of a feedback. This permits the correction and adjustment of the control parameters of the methods used. The originality of the suggested co-operation carries on the introduction of a mechanism, which checks the coherence of the results through a comparison of the two segmentations. From over-segmentation results, both methods are iterated by loosening certain constraints, until they converge towards stable and coherent results. This coherence is achieved by minimising a dissimilarity measure between the edges and the boundaries of the regions. The aim is therefore to provide the optimal solution in the sense of compatibility between the segmentation results. The system therefore uses a hybrid co-operation approach and is almost automatic and unsupervised. The performance of this approach has been measured on two remote sensing applications: agricultural landscape segmentation and forestry vegetation classification. (C) 2002 Published by Elsevier Science B.V.
\end{abstract}

Keywords: Image segmentation; Edge detection; Contrast perception; Region extraction; Methods co-operation; Dissimilarity measure between maps of edges

\section{Introduction}

The extraction of the different entities that compose an image constitutes a fundamental task in the image processing and analysis chain. This operation, called segmentation, is often obligatory in all artificial vision systems. It greatly affects the quality of results of the subsequent steps of the analysis. Although there is already vast literature on the subject [37,39,41,50], a general segmentation method, which performs well in many contexts, does not exist. The employed techniques remain generally dependent on:

- the specificity's of the image to process: richness in textures with different orientations and/or scales, blurred transitions between regions, occulted contours, etc.,

- the types of visual indices (primitives) to extract: edges (steps, lines, junctions), uniform regions in the sense of grey levels, textures, forms (segments, curves, etc.), etc.,

\footnotetext{
* Corresponding author. Tel.: + 33-296-46-5030; fax: +33-296-46-6675. E-mail addresses: chafik.kermad@irisa.fr (C.D. Kermad),

kacem.chehdi@enssat.fr (K. Chehdi)
}

- the nature of the problem to be solved below the segmentation: 3D reconstruction, pattern recognition, image understanding, automated object tracking, etc.,

- the exploitation constraints: computational complexity, real time functioning, materials constraints linked to the acquisition systems, memory capacity, etc.

In addition, as is the case with many tasks in computer vision, the image segmentation is an ill-posed problem in the sense of Hadamard [31,44]: the uniqueness, the certitude and the stability of the solutions relative to the data entries are not guaranteed:

- the application of different algorithms to the same image often gives different results, sometimes with a hugely variable redundancy;

- tiny modifications of the initial data or parameters of the method (thresholds, scale factors, sizes of analysis windows, pixel scanning directions, etc.) can produce important changes in the results.

The problem of segmentation still remains open, above 
all in the case of complex images that contain a large variety of uniform and/or textured regions.

In this paper, we propose an automatic and unsupervised system of image segmentation through iterative co-operation between methods. Therefore, the segmentation is obtained without a priori knowledge of the number, positions, sizes, models or shapes of the regions existent in the image, and without hypothesis on the distribution of the grey levels of the image. The proposed system is also capable of adapting the method's parameters to the image's specificity, so that it can be applied to multiple classes of images possessing regions of different nature.

Before we present the adopted approach for reaching these objectives in Section 3, in Section 2, we briefly expose the drawbacks of the main segmentation methods (non-cooperative and co-operative) presented in the literature. In Section 4, we measure the performance of the proposed system on two applications: agricultural landscape segmentation and forestry vegetation classification. In Section 5, we finally conclude and give some perspectives.

\section{Related works and motivations}

This section aims to illustrate the difficulties linked to the problem of segmentation that have never been efficiently solved by using the classic techniques. We then present the methods based on the combination of several techniques, focusing essentially on their weak points in order to take these into account in the design of the developed system.

In spite of the diversity of segmentation techniques, it is possible to classify them into two large categories: non-cooperative approaches and co-operative approaches. In the category of non-co-operative approaches, we can distinguish three types of methods: those based on the edge (local transitions) detection [30,45], those founded on the uniform region extraction [48] and those dedicated to the textured region extraction [39]. The application of these methods leads to the following remarks [22]:

- The edge detection methods generally suppose a priori model used to detect and operate in a very localised way. They often provide discontinued contours. Furthermore, they are sensitive to abrupt spatial variations of the image, caused by either the textures or by the presence of noise.

- The uniform region extraction methods principally introduce errors relative to the localisation of the boundaries of the regions.

- The least satisfying results of the textured region extraction methods are essentially due to the difficulties in the characteristic information extraction that emphasises the separation between the different models of textures. Moreover, in the majority, the texture segmentation methods suffer from the problem of the indefinite localisation of the regions' boundaries. They finally present the defect that alters the fine structures in the image, which are either often merged with the neighbouring regions or become over-segmented.

These comments show that it is difficult to obtain satisfying results of partitioning of an image using only one segmentation method. In order to overcome these difficulties, much work has had recourse to the co-operative approaches based on the combination and integration of several methods [34], especially those of edge detection and uniform region extraction [38]. As will be shown later, these two methods present some characteristics, which are complementary in many respects, in particular for the first method, the precision of the localisation of the edges and for the second, the resistance to noise and the natural closure of the regions' boundaries. Three schemes of co-operation can be distinguished: the sequential co-operation [5,16,21,25, $33,38,46,47]$, the parallel co-operation $[1,6,14,18,26,29,32]$ and the hybrid co-operation $[4,8,10,24,36,42,43,49]$.

- The different approaches of the sequential co-operation suffer from weak performances caused by the imposed order in the co-operation. What's more, in most of the approaches, the regions intervention level is different to that of the one of edges.

- Concerning the parallel co-operation approaches, and according to the specific case, the emphasis is mainly focused on the problems of fusion, adaptation or correction. The notion of active co-operation, which implies the simultaneous introduction of these three mechanisms, does not explicitly intervene.

- The approaches of hybrid co-operation alleviates the difficulties found in the first two categories of approach, nevertheless they do present the default, that is, linked to the lack of decisional criteria that allows the coherence validation of the extracted primitives.

In summary, the automation of the segmentation task and its adaptation to a large range of image types requires the recourse to several complementary methods together with the adoption of a progressive approach, where the primitives' construction operates in a co-operative and guided manner. In addition, the efficiency of co-operation can be further improved by introducing some criteria to the adaptation and the correction mechanisms that allow the evaluation of the degree of robustness of the different primitives extracted by the techniques implicated in said cooperation. Our work lies in this perspective and aims at refining and enriching the existing co-operative methods.

\section{Developed approach}

The main idea of the developed approach is based, firstly, on the fact that a common result to several segmentation techniques can be considered as significant, and secondly, 


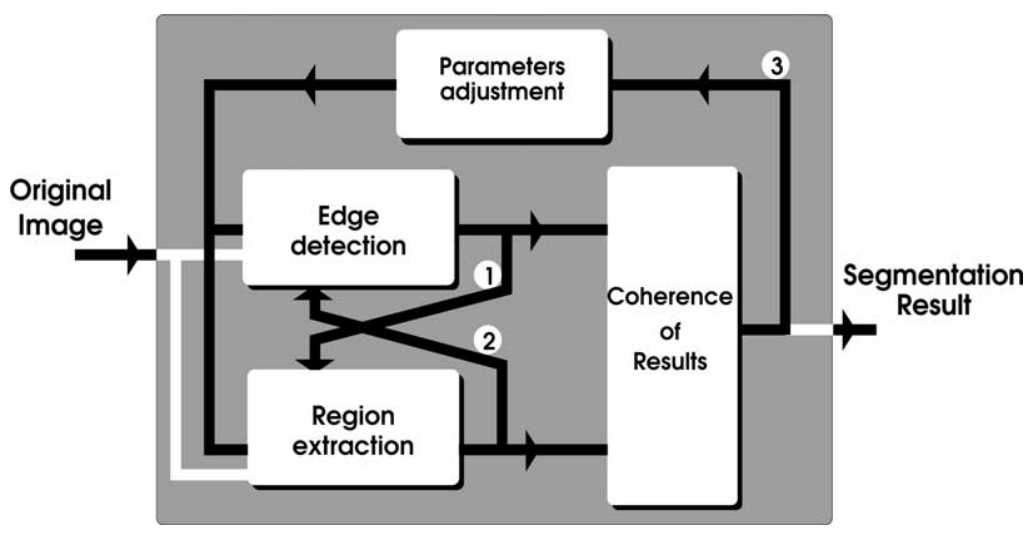

Fig. 1. The segmentation system synopsis.

on the complementary of the results to fulfil the weaknesses of each of the different segmentation methods. Moreover, to generate a coherent and stable image representation, a mechanism allowing the checking of the coherence of the extracted primitives is used. Finally, a feedback is introduced in order to adjust the control parameters of the segmentation methods. The current system is made to integrate two methods: an edge detector and a uniform region extractor. Its synopsis is shown in Fig. 1.

The following steps describe the global segmentation procedure. (i) Initialisation of the system by an overdetection of edges and an over-segmentation of regions (nuclei). (ii) Region growing around the nuclei under the control of the detected edges (lock (1) on the synopsis (Fig. 1)). (iii) Correction and refinement of the map of edges with the aid of the extracted regions (lock (2) on the synopsis). (iv) Transformation of the map of regions into an edges map and estimation of the coherence between this and the edge detection result. This estimation is performed by a dissimilarity measure, which takes into account the detection and the topology of edges and region boundaries. The segmentation procedure is iterated (from step (ii)), by loosening the formation criteria of regions (lock (3) on the synopsis), until the results are stable and coherent. The retained segmentation is the one, which minimises the dissimilarity (maximises the coherence) of the region's with the edge detection result [23].

In the following sections, we will firstly present the edge detection and the region extraction methods used in the system. Then, we will describe the interaction between these two methods.

\subsection{Adopted method for the edge detection}

From a preliminary study in Ref. [22] and seeing the remarks on different edge detectors in Refs. [11,50], we have chosen to use Deriche's approach [13]. In fact, the filter implemented through this approach is optimum in the sense of Canny's criteria [7], and it presents the property of being parameterable through a scale factor $\alpha$, which controls the degree of smoothing. The filter behaviour study, at different scales, shows that its application for the increased values of $\alpha$ (decreased scales) allows for better edge localisation to the detriment of a strong sensitivity to noise. The choice of an optimal scale remains an unsolved problem and an over-segmentation would have been preferable to an under-segmentation. An edge detection with an increased value of $\alpha$ is preferable [15].

After the filtering operation, the detection of edges can be accomplished in two ways: either by the first derivative local maxima extraction or through the second derivative zero crossing. In our case, the candidates for retained edge points are those, which correspond to the local maxima of gradients. This operation is generally followed by a hysterisis thresholding [7]. The advantage of this type of thresholding is that it will allow the obtention of some connected point groups. It, however, requires the knowledge of two parameters $S_{l}$ and $S_{h}$. For this operation, a compromise is often difficult to attain that conserves the edges corresponding to the significant region borders and eliminates the 'false edges' caused by noise.

In order to avoid the hysterisis thresholding, which imposes the fixation of the two thresholds and subsequently to favour an adaptive thresholding of the grey levels and the context of the examined neighbours, a classification of the gradient maxima into three categories (weak, medium and strong contrast) is achieved according to criteria that take into account human visual perception. For that, a function of Sensitivity of the Eye to the Contrast (SEC) integrating three levels: strong, medium and weak, has been determined [9]. We remind you of the principle of the function construction (for more details, see Ref. [27]).

To measure the SEC, we use the idea contained in the method proposed by Weber and Fechner [17]. A set of images composed of vertical bands is created. Each band in the image corresponds to a constant level of illumination. Commencing from the weak level of illumination (0) towards the increased level (255), two successive bands are differentiated by a contrast $C$. For a set of contrast values, a series of images is obtained. The observation of this series shows that three zones are generally distinguished. The extreme zones, corresponding to the weak and increased 


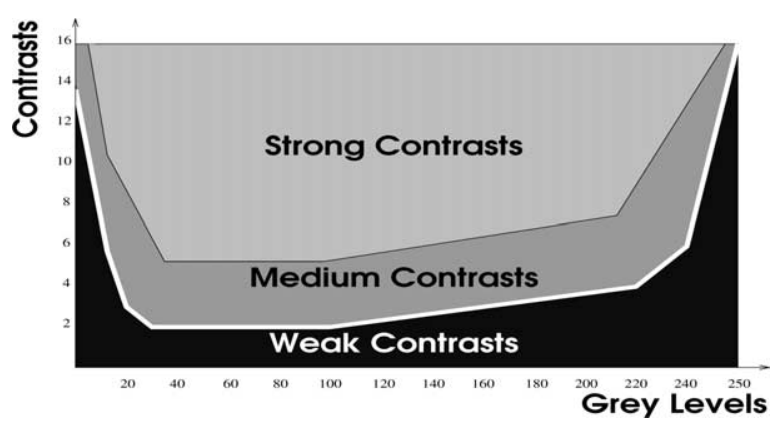

Fig. 2. Function of the SEC.

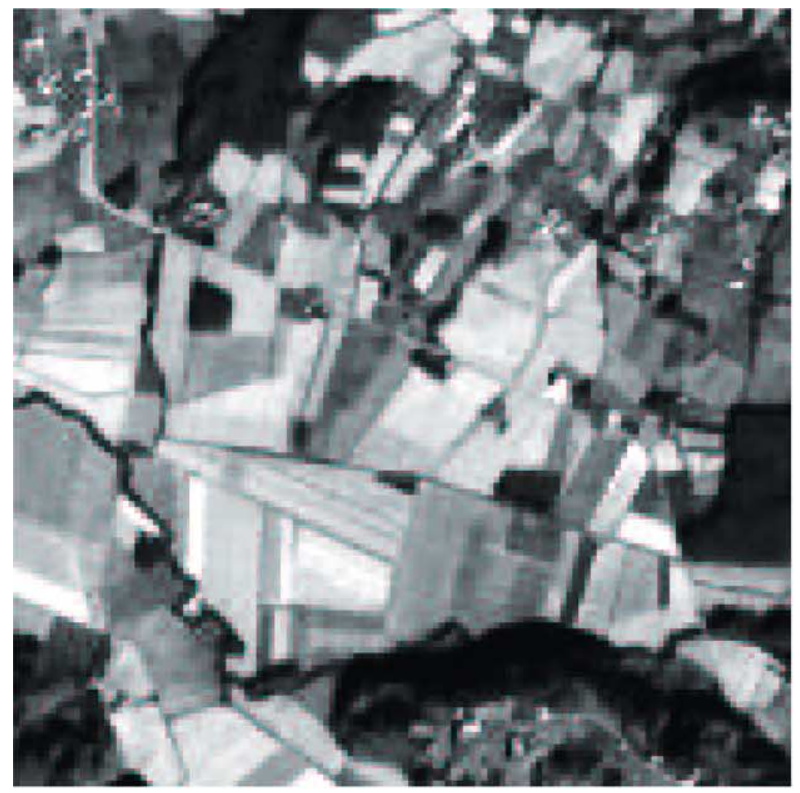

(a)

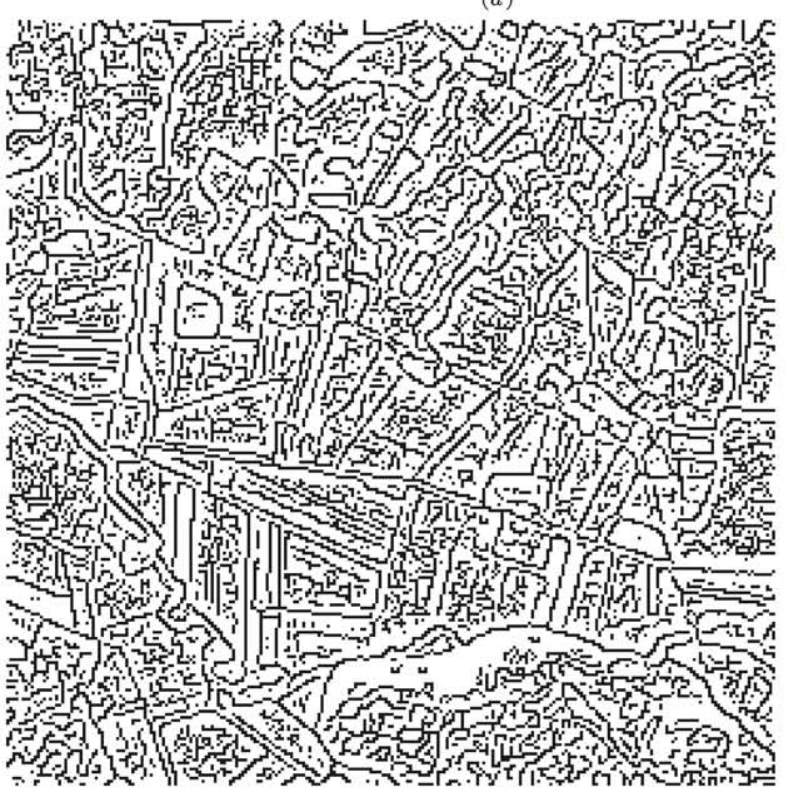

(c) illumination bands, are practically homogeneous. That means the borders between the bands of each zone are invisible to the human eye. On the other hand, the central zone bands are easily distinguished. The limit values of this central zone and the value of the contrast $C$ allows us to obtain a decision curve (in white in Fig. 2), which separates the visible contrasts from the invisible ones (weak contrasts). A finer analysis has been carried out to divide the visible contrasts into two areas: strong contrasts and medium contrasts [27] (Fig. 2).

The perception of the contrast between a pixel and its neighbours is dependant on its level of illumination and on

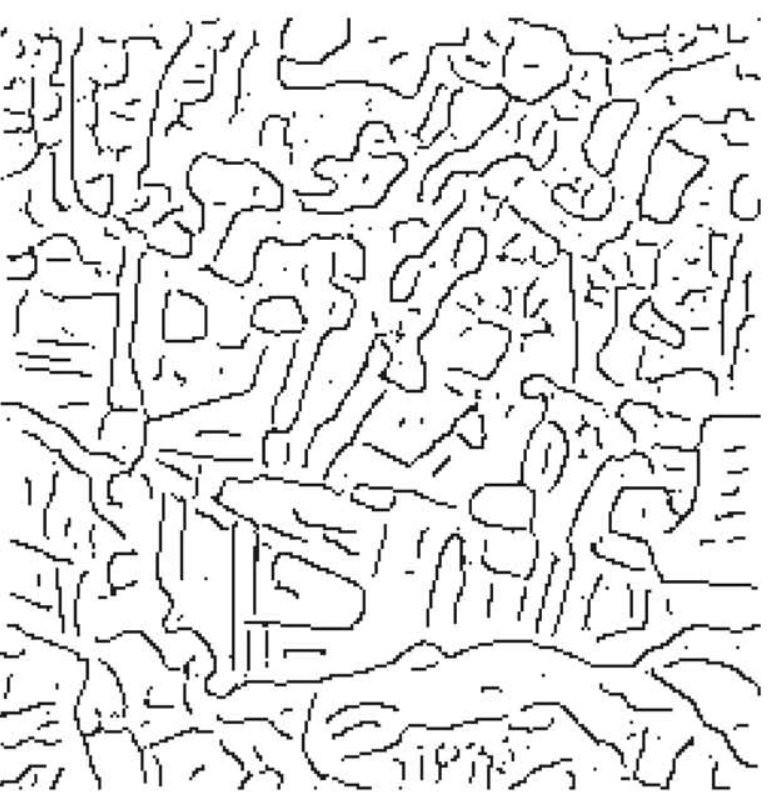

(b)

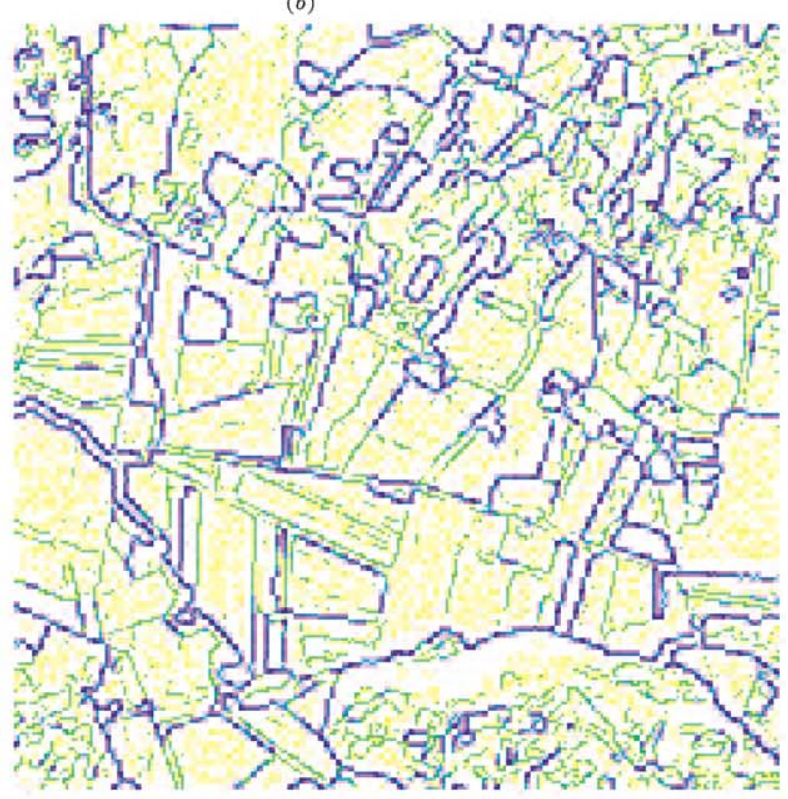

(d)

Fig. 3. Detection of the local maxima through the Deriche's filter: (a) SPOT image, XS3 canal, of the Aquitaine region after pre-processing (contrast enhancement). (b) Edges obtained at $\alpha=0.5$. (c) Edges at $\alpha=2$. (d) Classification of gradient maxima ( $\alpha=2$ ) into 3 classes (weak, medium and strong: darkest pixels). 
the differences of illumination with neighbouring pixels. The eye is least sensitive to the transitions in the increased illuminations (very clear zones of the image) and the weak illuminations (darkened zones). For example, for the contrast to be visible, a pixel having a grey level equal to 10 must have a gradient of 8 in relation to its neighbouring region. But, it must only have a gradient of 2 if this pixel has a grey level equal to 80 . The SEC function allows us to class the edge points by associating them with confidence indices (strong, medium or weak). These indices are determined by the position of the grey level and the gradient from the point in the three zones of the function. The confidence indices associated with the edges will be used to guide and assist the formation of the uniform regions. These will then be used to validate the significant edge points (cf. Section 3.3).

To illustrate Deriche's operator's behaviour and the SEC function utility, we present results obtained for a remote sensing of an image taken by the SPOT satellite ('Aquitaine' image, Fig. 3(a)) issued from the CNRS PRC-ISIS image bank. The images (b) and (c) in Fig. 3 show examples of the edges extracted from the Aquitaine image using two different values of $\alpha$. In these results, the local maxima correspond to black pixels and the rest to white pixels. We can note that the decrease of $\alpha$ favours a significant detection to the detriment of a good localisation and vice versa.

The image (d) of Fig. 3 shows the classification result of the image (c) (gradient maxima corresponding to an $\alpha$ equal to 2). The darkest pixels correspond to the strongest gradients. This result shows that the SEC function enables us to easily distinguish the edge points that correspond to the fluctuations of grey levels in uniform regions (weak contrasts represented by brightest pixels) from those linked to transitions between uniforms regions (darkest pixels).

\subsection{Adopted method for the region extraction}

The regions formation can be realised in two different ways: (i) split and/or merge process founded on some global criteria, (ii) a points aggregation procedure based on some local similarity criteria. Due to the global level of the analysis, in a split and merge process the local information is not considered. On the other hand, a points aggregation to a region needs to take into account some global information of the region, as well as some local information relative to the pixel. For this reason, the region extraction through points aggregation approach has been retained.

The nuclei of regions are first determined, then their characteristics are calculated. Neighbouring points to the nuclei are then localised. Among these points, only those that verify a similarity criterion are aggregated to the nuclei. If a point is adjacent to more than one nucleus, it will be annexed to the one with which it has the strongest degree of association (in the sense of the employed similarity criterion).

In general, the regions' nuclei are introduced by a user.
In the unsupervised case, the reliable nuclei can be determined by logical operations between different region segmentations of the image [21]. They are therefore obtained by the intersection of many maps of the regions. These results are carried out through the same region growing procedure but by using different scanning directions (image scanning line by line and column by column) and by applying weak segmentation thresholds to guarantee the region's homogeneity. This is the approach that we have used.

The vector of characteristics of the nucleus or the region constitutes of its mean intensity, its standard deviation and the number of its pixels, noted $N_{R}$. At commencement, the model of the region is initialised by the nucleus parameters. During the course of the region expansion, when a pixel is added to a region, its model is then updated. According to whether or not the estimate takes a recursive form two approaches for updating the parameters are possible. With a recursive estimation, it is not necessary to manipulate all data in order to calculate the new value of the parameters. From this fact, this approach is of interest to image segmentation, when the regions contain a large number of pixels. It allows a noticeable reduction of processing time.

The empirical estimates of the average and the standard deviation for the $\left\{x_{(n+1)}\right\}$ first observations can take a recursive form as follows [19]:

$\hat{\mu}_{x_{(n+1)}}=\frac{n}{n+1} \hat{\mu}_{x_{(n)}}+\frac{1}{n+1} x_{(n+1)}$
$\hat{\sigma}_{x_{(n+1)}}^{2}=\hat{\sigma}_{x_{(n+1)}}^{2}-\left(\hat{\mu}_{x_{(n+1)}}\right)^{2}$

where

$\hat{\sigma}_{x_{(n+1)}}^{2}=\frac{n}{n+1} \hat{\sigma}_{x_{(n)}}^{2}+\frac{1}{n+1} x_{(n+1)}^{2}$

To measure the degree of resemblance of the attribute (the intensity $I(i, j))$ of the examined pixel with the model of the region in the course of construction, the similarity criterion corresponding to the Fisher's test is used. An examined pixel of intensity $I(i, j)$ is aggregated to the region $R_{c}$ with a $\mu_{R_{c}}$ average and a $\sigma_{R_{c}}$ standard deviation if

$\frac{\left|\mu_{R_{c}}-I(i, j)\right|}{\sigma_{R_{c}}} \leq S_{k}$

where $S_{k}$ is a threshold, which represents a parameter of the system. It is initialised with a weak value and then increased at the end of each iteration $k$ of the system. The regions' nuclei or regions extracted at each iteration must also to have a number of points superior to a threshold $N_{R_{k}}$. This parameter is equally re-actualised at the end of each iteration of the system.

Note that in the aggregation process, some small pixel groups, which do not verify this criterion, can exist. These pixels often situated on a discontinuity or even on boundaries of this region, have some local characteristics, which are not the same as those in the neighbouring region. 


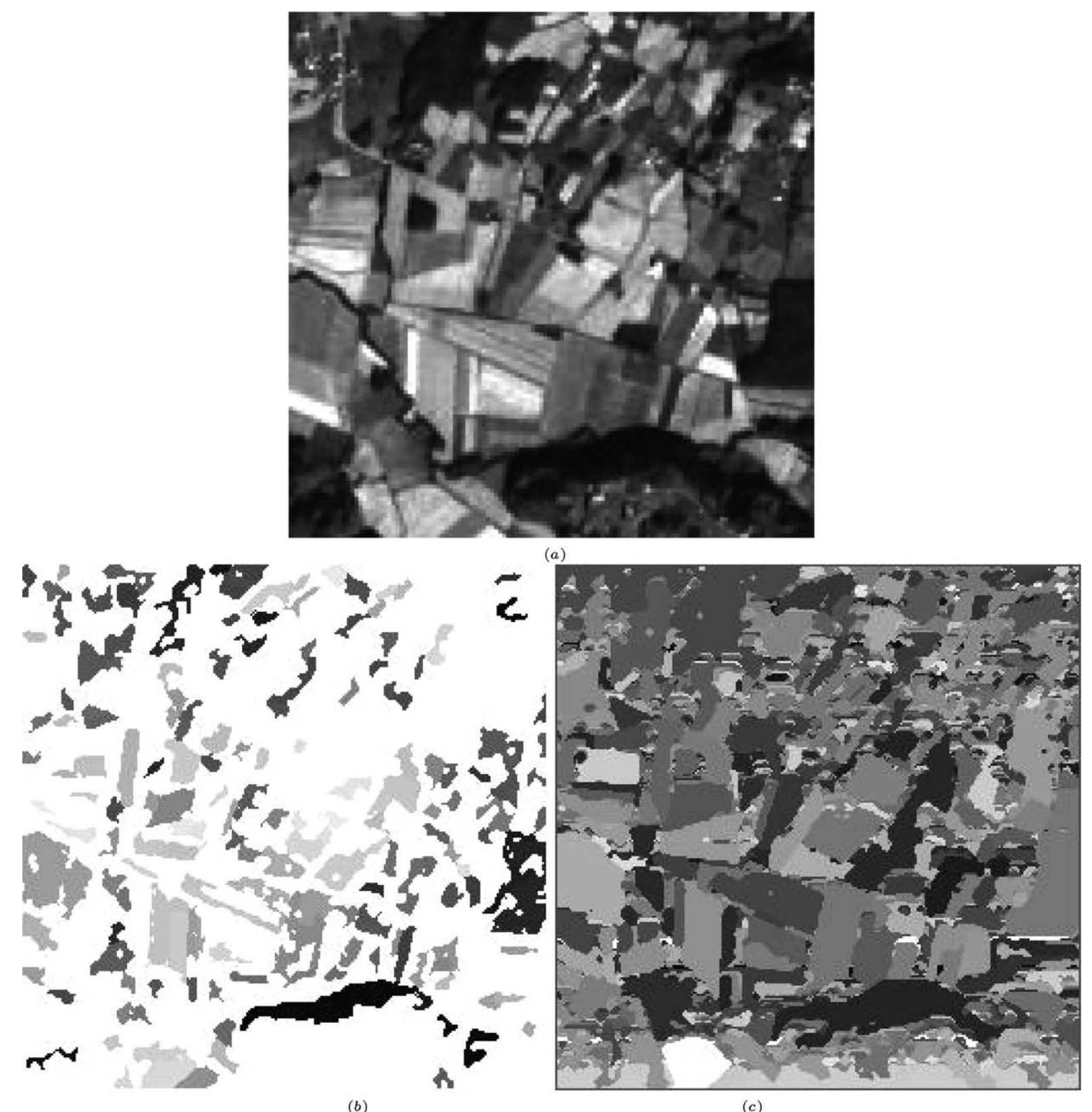

Fig. 4. Extraction of the regions through recursive points aggregation: (a) SPOT image of the Aquitaine. (b) Nuclei produced with an $S_{k}=5$. (c) Regions obtained from (b) with $S_{k}=5$.

The annexation of such pixels will imply an arbitrary variation of the region's average and standard deviation. It is therefore hoped to class them in a category of problematic pixels and to retard the decision of their annexation to such and such a region until some information resulting from other processes (edge detection, textures extraction, etc.) can be obtained.

Fig. 4 shows an example by applying the recursive points aggregation algorithm to the SPOT image of 'Aquitaine's' (Fig. 4(a)). The nuclei (Fig. 4(b)) are obtained through the intersection of four segmentation results carried out by the points aggregation algorithm using four scanning directions (line by line from top to bottom, and line by line from bottom to top; column by column from left to right, and column by column from right to left). The four segmentation results are obtained by using the same value of threshold $\left(S_{k}=5\right)$. The regions of Fig. 4(c) are obtained by aggregation of points around these nuclei with an $S_{k}=5$.

This result shows that in spite of the use of weak similarity thresholds, certain regions of the image are undersegmented. We can equally note the relatively poor localisation of the majority of the extracted boundaries of the regions.

The methods used to extract the image's meaningful regions assume the uniformity of the regions of interest. Therefore, the aims of the different techniques are often to 
separate the image by respecting many arbitrary proprieties that are strongly related to the region's homogeneity. This restrictive hypothesis is at the base of some principle failings of segmentation algorithms for region extraction (the difficulty to correctly detect the position of the boundaries of the regions). Therefore, a region where the luminosity varies linearly can be divided into two subregions and two distinct regions that have some weak contrasts on the length of their borders can thus be merged into one. In order to assist the region extraction technique, this is one of the reasons, which make it necessary to recourse information issued from the edge detection.

\subsection{Edge-region co-operation and interaction}

Ideally, the description of an image from edge and region primitives must be identical (a closed contour defining a region's boundary, a region's boundary defining a closed contour). In practice, the differences are important and we rarely obtain equivalent descriptions from these two primitives. Typically, as mentioned earlier, the principal advantage of the edges are that they are localised in a precise manner. Nevertheless, the application of an edge approach often coincides with a problem of under-detection of certain discontinuities, which create open contours. The strong point of the region extraction approach is the closure of their boundaries and the richness of the information that they convey. However, their exact localisation remains difficult to obtain.

The adopted methodology in the developed system tends to gain from the complementary nature and the compensation between the uniform regions and the edges in the image. The co-operation is concretised by the extraction of a type of information guided and/or corrected by the dual one. This duality and complementary can be expressed in four different ways $[6,21,23,36,38,47]$ :

- the regions are situated in the interior of close contours and consequently there are no edge points in the interior of a region;

- a real edge point must be situated on or at the proximity of a region's boundary;

- a region's boundary is naturally close and an edge must be equally close;

- an edge cannot be situated in the interior of a region and must be situated on the totality of the common border between two regions.

On the basis of these rules of duality, a certain number of decisional criteria are used to correct the edges and the regions obtained at the issue of the different iterations of the system.

\subsubsection{Edges refining using the extracted regions}

To eliminate the false edges linked to the presence of noise and to confirm those that are significant, the edge detection process exploits the information from the regions. This information is generally more global. The edges can be refined and the detection can therefore be more reliable. An edge point is validated if its gradient belongs to the class of strong contrast gradients. It is rejected if the regions that border it have the same labels and if its gradient belongs to the class of the weak contrast gradients. On the other hand, if the edge point belongs to the class of medium contrast gradients, it is validated when the regions situated on either side of the edge possess different labels and at least one of its neighbouring points has a gradient belonging to the strong contrast class.

Another aspect of the edge-region interaction is related to the closure of edges. This consists of filling in the discontinuities between a series of points that represent the same contour. The closure of edges is generally initialised by a perceptual regrouping. The first stage consists of examining the neighbouring chains of edge points by applying the Gestalt criteria [28]. These criteria are generally given through

- proximity: two chains cannot be merged if their distance is superior to a threshold;

- continuity: the contour often being rectilinear, two chains have a strong probability of merging if they are in the prolongation each from the other.

Once the regrouping has been decided, each pair of the edge point chains concerned is connected by a curve, defined with or without photometric criteria characterising the hole separating the chains. The curve, which joins two chains of edge points, is defined either by using a model of curve (right segments, circle arcs, polynomials, etc.) or by minimising a function of energy of curving. The closure is founded on local criteria of the two chains [35] or on a global optimisation strategy [2,12]. Our current closure implantation is simple. Two chains of edge points, situated on or in proximity of a region's boundary, are connected by a curve that aligns with the local gradient maxima if the distance that separates them does not exceed three points and if their extremities possess the same gradient direction. An unconnected edge, included in the same region, is conserved if it belongs to the class of strong contrast gradients. This kind of edge reflects some defects relative to the object's surface state (textures having strongly contrasted patterns) or to the presence of shadows or reflections. Therefore, it is possible that an edge stays in the interior of a region.

\subsubsection{Regions' boundaries correction using the detected edges}

Furthermore, as previously mentioned, in addition to an imprecise localisation of boundaries, the region extraction procedure creates some under-segmentations in spite of the use of weak thresholds. The unsuccessful localisation of borders is mainly due to the strict definition of the 
uniformity of the region. The errors relative to the problem of under-segmentation are principally caused by the noise created by pixels superimposed on the boundaries and possessing similar grey levels to the neighbouring regions. Also, to partially overcome this default, a second criterion has been introduced in the procedure of points aggregation that makes the gradient of the examined point intervene. In addition to the employed similarity criterion, the examined point has to have a gradient that belongs to the class of weak contrast gradients in order to be aggregated to the region.

Remembering that the researched aim in this system is to provide some mutual and compatible results in order to obtain a coherent segmentation. The coherence is based on the assumption that a description of an image from edge and region primitives must be identical. It is carried out by minimising a dissimilarity measure between the edges and the boundaries of the regions.

\subsubsection{Coherence of results by minimising a dissimilarity measure}

Given many segmentations of the same scene, the problem here, is to find a measure, which allows us to give an estimation of the similarity, or the correlation between the elements of the different segmentations, by at the same time considering both the detection and localisation of the different extracted primitives. In order to define a common representation of information to put into correspondence, a transformation of the different results is first carried out. The measure of dissimilarity between the transformed results is then effectuated. For reasons of simplicity and calculation time, a representation in the form of edges has been adopted. Note that a modelling of highest level (based on geometrical primitives, such as straight segments or others) will be more significant and reliable in obtaining better results for a slightly textured environment presenting strong discontinuities. However, they will need to use interpolation techniques and more complex similarity measuring methods, which create a more important computational time.

Through the measures that allow us to give an indication of the dissimilarity between edges, we can cite the Hausdorff distance [20]. The Hausdorff distance between two subsets $A$ and $B$ belonging to a set of points $X$ is given by

$D_{\mathrm{H}}(A, B)=\max \left\{\sup _{x \in A} d_{E}(x, B), \sup _{x \in B} d_{E}(x, A)\right\}$

where if $d$ is a distance between two points, then the distance $d_{E}(x, A)$ between a point $x$ and a group of points $A$ is defined by

$d_{E}(x, A)=\inf \{d(x, a)$ pp $a \in A\}$

Although the Hausdorff distance is theoretically interesting as it possesses some properties that relate to the basic operations of mathematical morphology [20], in practice it is rarely used for error measuring between edge maps [3].
The inherent problem with this distance is above all due to its great sensitivity to noise, which will produce false edges. A simple pixel, that is, badly located increases the value of $D_{\mathrm{H}}$ because of the presence of the operator 'sup' in its definition. To erase this sensitivity of $D_{\mathrm{H}}$ to noise and to take into account its interesting proprieties of topology, we propose the use of a measure approaching an averaged Hausdorff distance. In the following, we describe this measure.

Let $C$ be the map of edges and let $F$ be that of the boundaries of the regions. The adopted approach consists of calculating, for each edge (resp. boundary) point in the image $C$ (resp. $F$ ), the minimal deviation, which separates it from a boundary, $d_{E}(c, F)$, (resp. from an edge, $d_{E}(f, C)$ ) point in the map $F$ (resp. $C$ ). The averages of the minimal deviations are then calculated for each map. The final dissimilarity measure corresponds to the overall average of two averages. It is given by

$\operatorname{Dissim}(C, F) \equiv 1 / 2\left\{\operatorname{Avg}_{c}\left[d_{E}(c, F)\right]+\operatorname{Avg}_{f}\left[d_{E}(f, C)\right]\right\}$

where $\operatorname{Avg}_{c}\left[d_{E}(c, F)\right]$ (resp. $\operatorname{Avg}_{f}\left[d_{E}(f, C)\right]$ ) is the average of the minimal deviations $d_{E}(c, F)$ (resp. $d_{E}(f, C)$ ).

This algorithm gives good results: it presents a strong robustness to the noise in comparison with the Hausdorff distance, and does not need an empirical threshold. The computational complexity of this measure depends on the number of edge points and the number of region boundary points in the image. This complexity remains nevertheless expensive in processing time in the case of detail rich images. To partially overcome this problem, we have reduced the research space of the closest point from $p$ in $M$ $(M=C$ or $F)$ to some limited and neighbouring regions centred around $p$.

\section{Experimental results}

To assess the performances of the developed cooperative approach, in this section we will present, three experimental results that correspond to two application examples: agricultural landscape segmentation and forestry vegetation classification.

In all these experiments, the region growing parameter $S_{k}$ starts at the value of 1 and increases in increments of 1 at each iteration. The regions surface parameter $N_{R_{k}}$ is initialised at 32 and decreases by a step of 1 . The chosen edge detector scale factor $\alpha$ has been fixed at 2.0 .

\subsection{Agricultural landscape segmentation}

In this experiment, we have tested the developed approach on two real images: a weakly textured image, as well as a strongly textured one. 


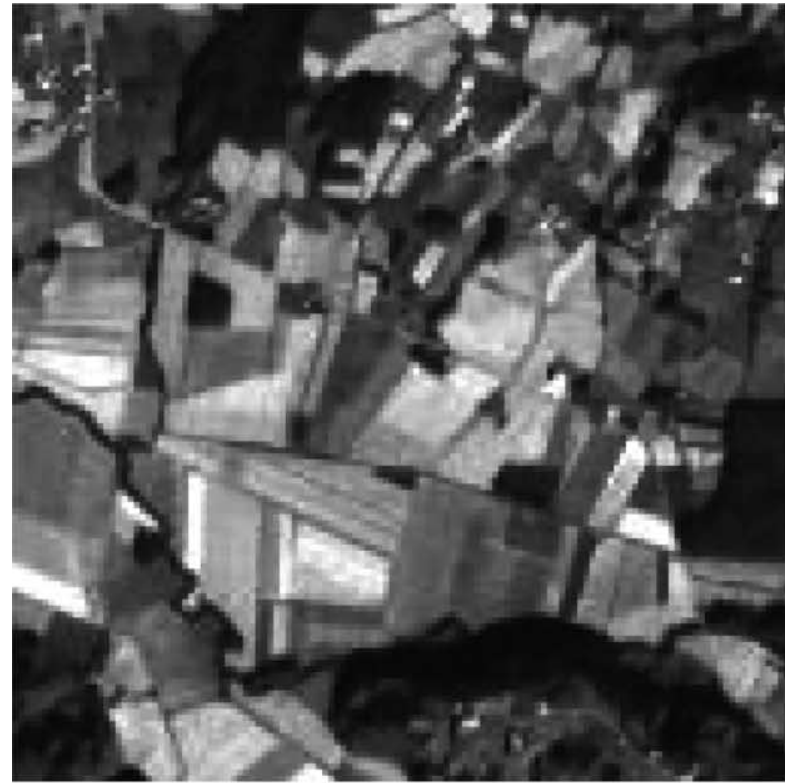

(a)

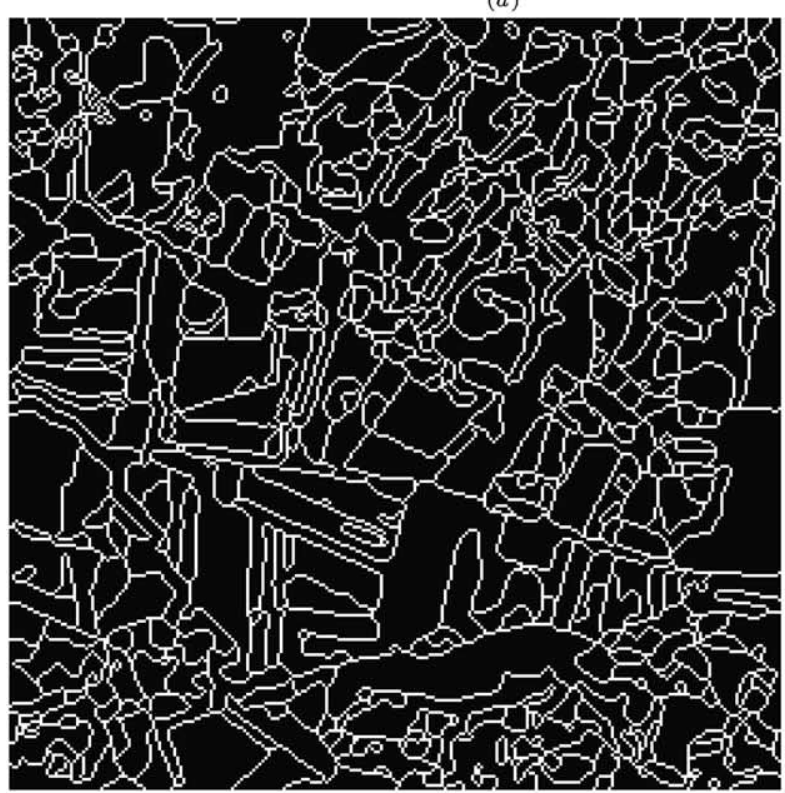

(c)

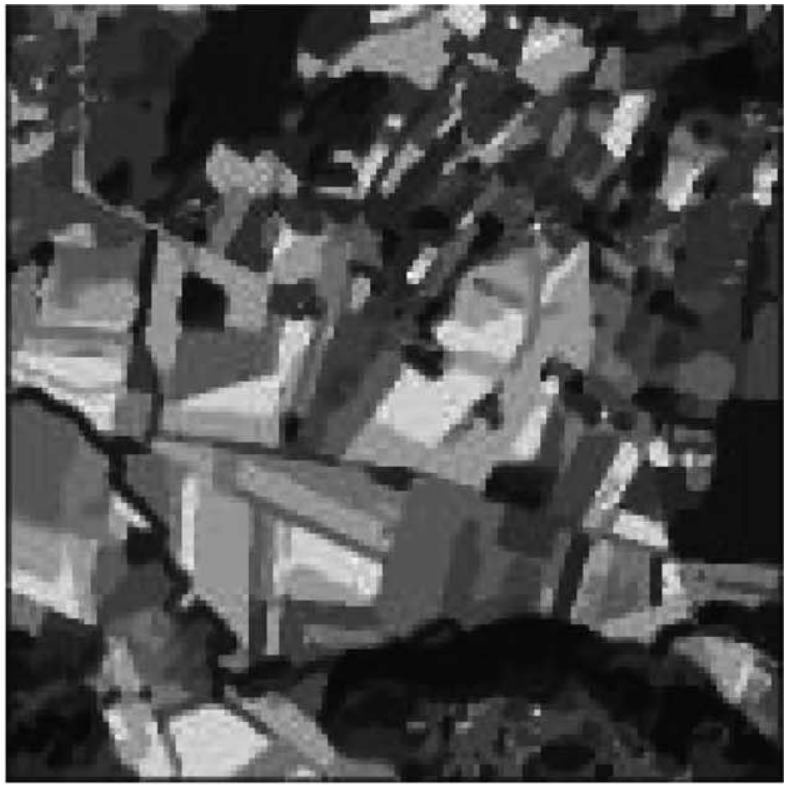

(b)

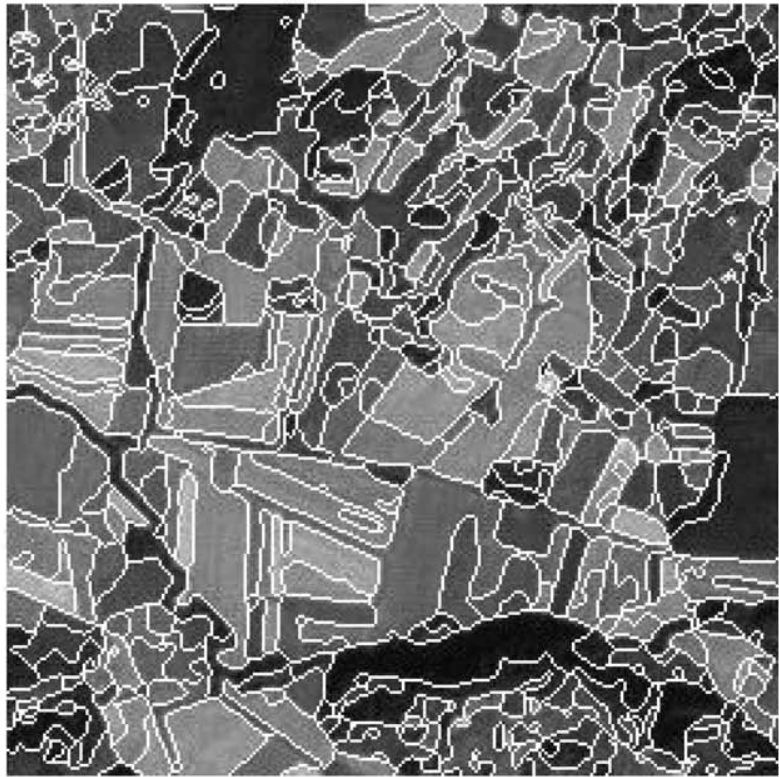

(d)

Fig. 5. Edge-region co-operative segmentation: weakly textured image: (a) Aquitaine original image. (b) Final result of the region segmentation. (c) Boundaries of the regions. (d) Boundaries superposed on the original image.

\subsubsection{Weakly textured image}

The images in Fig. 5 represent the segmentation results issued from the Aquitaine SPOT's image. We remark that a good segmentation is obtained. The majority of the visible original image regions are detected in a satisfactory manner.

The final edges correspond well to perceptually valid discontinuities in the image. Most of them are correctly extracted. We can equally note their good localisation and the preservation of the fine structures.

The curve in Fig. 6 illustrates the evolution of dissimilarity measure as a function of the iterations. This evolution shows that the segmentation result begins to stabilise from the seventh iteration.

\subsubsection{Strongly textured image}

The second tested image has been issued by the EPFL images bank. It represents an aerial scene taken by a CCD camera. This image contains rich textures in different orientations and presents some considerable non-homogenous regions (Fig. 7(a)).

In examining the segmentation result (Fig. 7(b)), in the sense of the grey level, we can state that, the different regions, which compose this image, have been well detected. Their boundaries align closely with the visible transitions in the image and the details are not lost. However, this result presents a multitude of small regions that are difficult to exploit. The segmentation method relies 


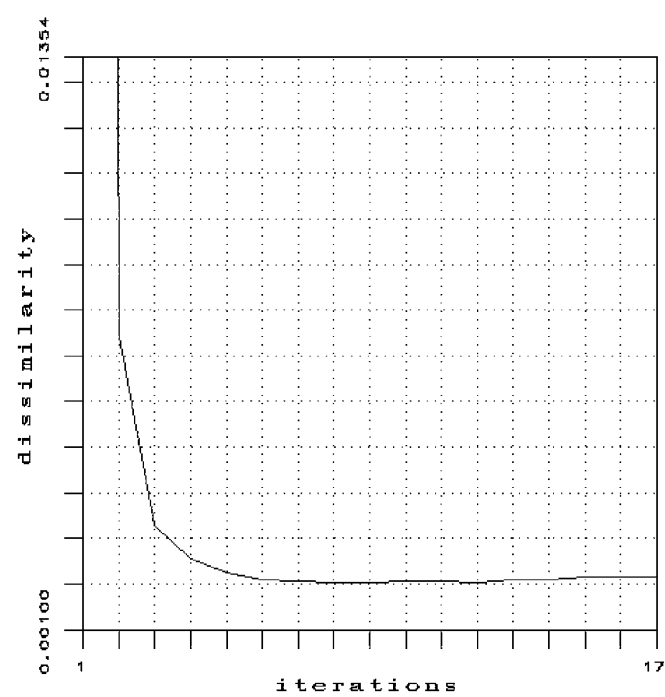

Fig. 6. Edge-region co-operative segmentation: aquitaine image. Evolution of the dissimilarity measure between the edges and the regions' boundaries as a function of iterations.

on the integration of other types of information based on the texture.

\subsection{Forestry vegetation classification}

In order to test the effectiveness of the developed method in a practice case, another remote sensing image taken by the SPOT satellite in July 1987, representing the Paimpont forest in Brittany (France) was used.

At our disposal for this image, we have the ground truth map, that is, composed of 12 classes and carried out by the COSTEL ('Climat et Occupation du Sol par TELédétection') laboratory at the Rennes II University France [40].

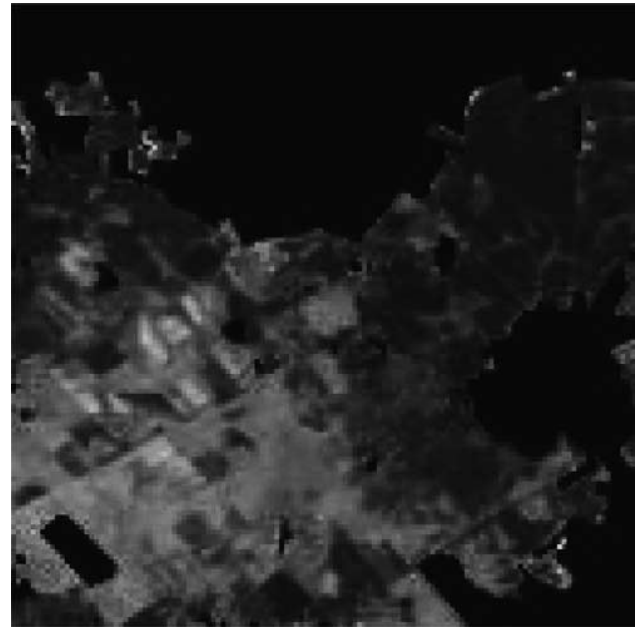

Fig. 8. Paimpont forest: masked CNES-SPOT image-XS3 canal.

As the ground truth map does not entirely cover the satellite image, this last has been masked so both have the same support (Fig. 8).

Table 1 lists the 12 thematic classes and the associated statistics (the surface, the mean and the variance values).

The image in Fig. 10(a) shows the superimposition of the ground truth map contours on the original image. The image in Fig. 10(b) shows the segmentation result obtained by the developed approach, superimposed on the original image.

On the whole, we can state that the borders of the visible regions in the original image have been well detected, their localisation is correct and therefore allows the envisaging of an interpretation process in a direct manner.

In order to classify the regions obtained from the image in Fig. 10(b), the modelling was carried out by, respectively, using the co-occurrence attributes (energy, inertia,

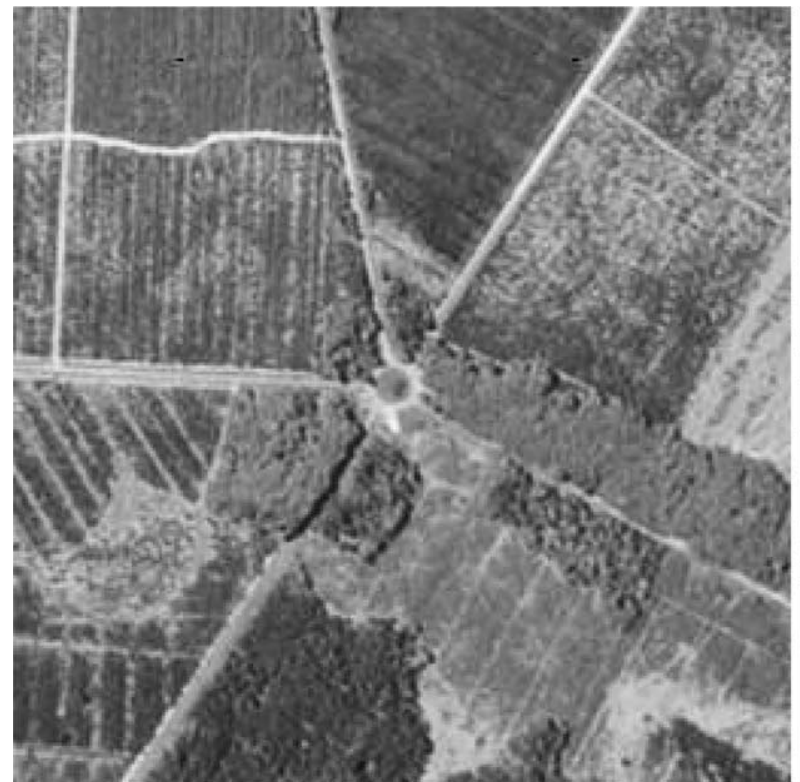

(a)

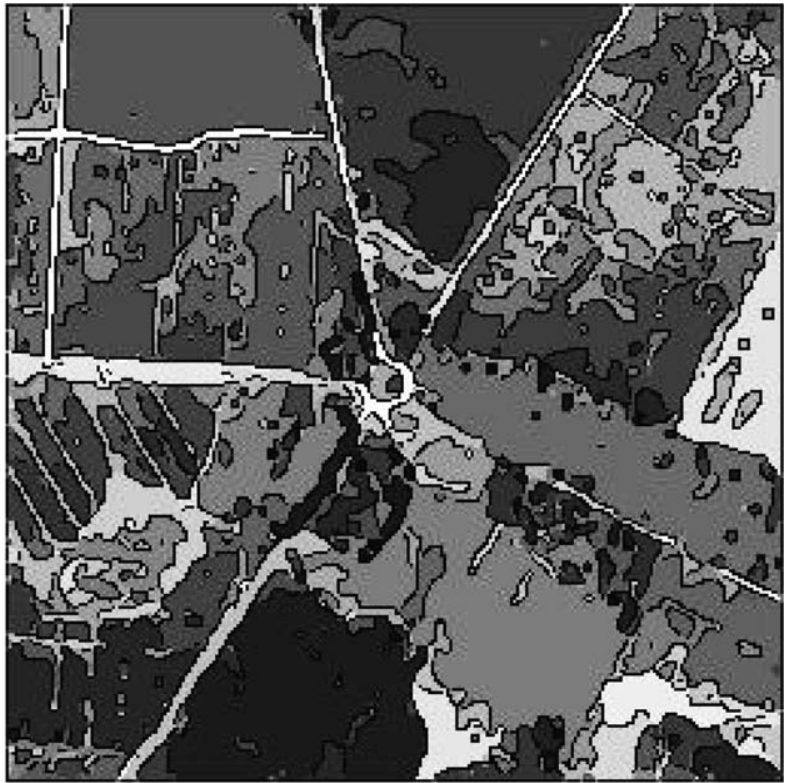

(b)

Fig. 7. Edge-region co-operative segmentation: strongly textured image. (a) Original aerial image. (b) Final boundaries superimposed on the regions. 
Table 1

Paimpont forest: Statistics of the 12 ground truth classes

\begin{tabular}{|c|c|c|c|c|}
\hline Class no. & Mean & Variance & No. of pixels & Thematic classes type of vegetation \\
\hline 1 & 69.5 & 15.51 & 1160 & Conifers, natural regeneration, sparse forest \\
\hline 2 & 76.9 & 18.61 & 5250 & Conifers, natural regeneration, dense forest \\
\hline 3 & 86.7 & 18.71 & 1372 & Conifers, natural regeneration, heterogeneous forest \\
\hline 4 & 91.9 & 23.49 & 1706 & Wooded heath-land \\
\hline 5 & 95.4 & 21.62 & 2672 & Conifer reserve \\
\hline 6 & 113.7 & 18.93 & 1922 & Conifers, artif. regeneration, directly sown plantations \\
\hline 7 & 118.9 & 23.14 & 1237 & Completely felled \\
\hline 8 & 125.1 & 35.48 & 3305 & Conifers, artificial regeneration, directly sown plantations, copse \\
\hline 9 & 134.8 & 26.45 & 10,542 & Brick thicket, copse \\
\hline 10 & 139.4 & 22.20 & 3193 & Broad-leaf reserve \\
\hline 11 & 139.7 & 14.09 & 648 & Broad-leafs, forest \\
\hline 12 & 145.0 & 21.88 & 489 & Broad-leafs, tall thickets \\
\hline
\end{tabular}

correlation, homogeneity and entropy extracted for displacements $(0,1)$ and $(1,0))$, the run-length attributes and the two first-order moments [39]. A $K$-nearest-neighbours classifier was employed using a Mahlanobis distance to perform the classification. Let $\left\{\Omega_{1}, \Omega_{2}, \ldots, \Omega_{N}\right\}$ be the set of models (attribute vectors) of $N$ regions issued from the segmentation. A region $R_{l}$ characterised by the vector $\Omega_{l}$ is assigned to the region $R_{i}$ modelled by $\Omega_{i}$ if $D\left(\Omega_{l}, \Omega_{i}\right)=$ $\arg \min _{j \in[1, N]}\left\{D\left(\Omega_{l}, \Omega_{j}\right)\right\}$, where $D$ is the Mahlanobis distance defined in the space of the models.

The images (b)-(d) in Fig. 11 represent the results after modelling and classification of the regions into 12 classes.

In order to be able to make an objective and quantitative comparison of the quality of the different classifications, the rates of identification have been calculated with the help of the ground truth map (Fig. 9(b)).

Tables $2-4$, respectively, give the rates corresponding to each of the three modelling methods. Here the identification rate represents, the percentage of the pixels of the $m$ th class (arabic numeral, first column) in the ground truth map assigned to the $n$th class (roman numeral) of the classification result.

In examining the identification rates obtained by the cooccurrence modelling (Table 2), we can note that the ground truth classes 1 and 2 (corresponding to the thematic classes of conifers, natural regeneration, sparse forest and dense forest (Table 1)) are assigned to class I, which is not completely incorrect and can be confirmed, when we examine the original image's grey levels (Fig. 10(b)). The ground truth classes 10 and 12 (associated to the thematic classes of broad-leaf reserve and tall thickets) are assigned to class XI and merged with the ground truth class number 9 (corresponding to the thematic classes of brick thicket and copse), this is also acceptable, when we look at the

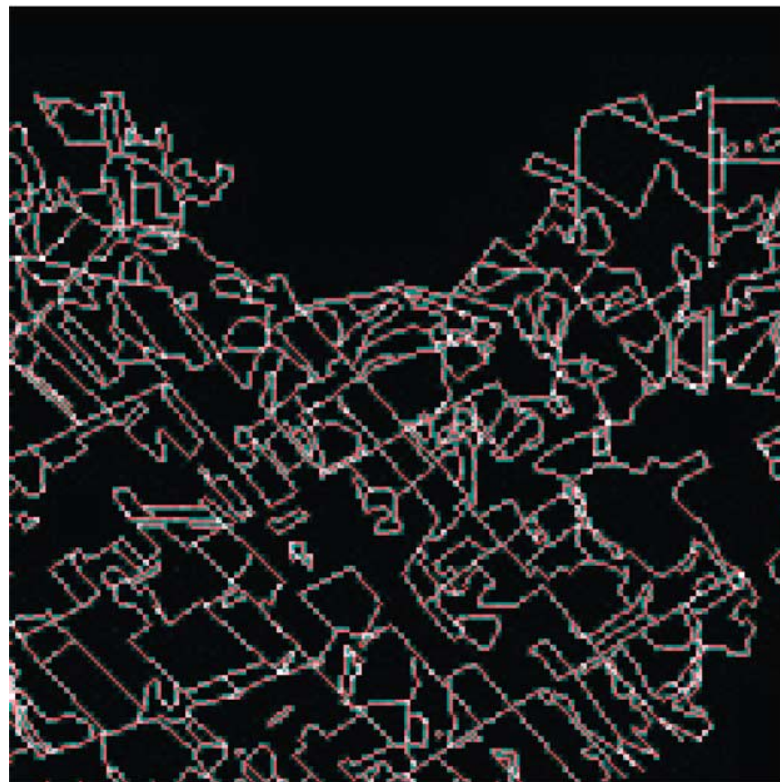

(a)

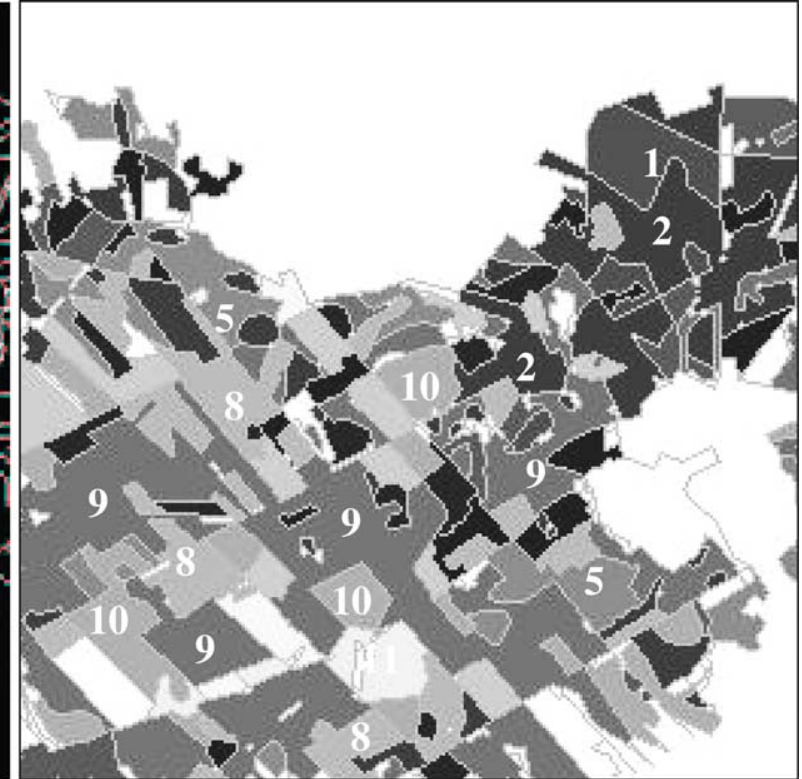

(b)

Fig. 9. Paimpont forest: ground truth map composed of 12 classes: (a) In contours. (b) In regions (with the class number for certain important regions). 
Table 2

Identification rate (in \%) between the classes of the ground truth (first column) and the classes issued from the co-occurrence modelling (first line)

\begin{tabular}{|c|c|c|c|c|c|c|c|c|c|c|c|c|c|}
\hline & I & II & III & IV & V & VI & VII & VIII & IX & $X$ & XI & XII & Other $^{\mathrm{a}}$ \\
\hline 1 & 80 & 2 & 1 & 0 & 1 & 0 & 0 & 0 & 0 & 0 & 0 & 0 & 16 \\
\hline 2 & 55 & 26 & 3 & 2 & 1 & 0 & 1 & 0 & 1 & 0 & 0 & 0 & 11 \\
\hline 3 & 25 & 31 & 19 & 5 & 3 & 0 & 1 & 0 & 0 & 1 & 0 & 0 & 15 \\
\hline 4 & 17 & 42 & 7 & 5 & 6 & 1 & 0 & 0 & 4 & 2 & 1 & 0 & 15 \\
\hline 5 & 12 & 41 & 13 & 9 & 2 & 0 & 4 & 1 & 3 & 1 & 2 & 0 & 12 \\
\hline 6 & 1 & 9 & 6 & 34 & 3 & 0 & 6 & 15 & 13 & 0 & 5 & 0 & 8 \\
\hline 7 & 2 & 9 & 10 & 23 & 6 & 0 & 3 & 6 & 8 & 8 & 10 & 1 & 5 \\
\hline 8 & 7 & 15 & 6 & 4 & 4 & 0 & 8 & 2 & 9 & 8 & 16 & 12 & 9 \\
\hline 9 & 2 & 5 & 5 & 5 & 5 & 0 & 3 & 4 & 6 & 8 & 45 & 2 & 10 \\
\hline 10 & 1 & 4 & 3 & 3 & 2 & 0 & 5 & 4 & 8 & 11 & 39 & 7 & 13 \\
\hline 11 & 0 & 3 & 1 & 0 & 0 & 0 & 0 & 8 & 3 & 57 & 26 & 0 & 2 \\
\hline 12 & 0 & 0 & 7 & 2 & 0 & 0 & 0 & 10 & 12 & 3 & 52 & 0 & 14 \\
\hline
\end{tabular}

a This class corresponds to a 'reject class', which essentially contains the pixels belonging to the boundaries of regions.

Table 3

Identification rate (in \%) between the classes of the ground truth (first column) and the classes issued from the run-length modelling (first line)

\begin{tabular}{|c|c|c|c|c|c|c|c|c|c|c|c|c|c|}
\hline & I & II & III & IV & $\mathrm{V}$ & VI & VII & VIII & IX & $X$ & XI & XII & Other \\
\hline 1 & 0 & 0 & 0 & 6 & 4 & 1 & 0 & 0 & 69 & 0 & 5 & 5 & 10 \\
\hline 2 & 0 & 1 & 1 & 13 & 2 & 9 & 6 & 10 & 35 & 4 & 8 & 3 & 8 \\
\hline 3 & 0 & 4 & 18 & 3 & 5 & 0 & 12 & 5 & 17 & 12 & 9 & 4 & 11 \\
\hline 4 & 0 & 2 & 1 & 4 & 7 & 10 & 5 & 7 & 15 & 20 & 11 & 9 & 19 \\
\hline 5 & 0 & 1 & 6 & 6 & 8 & 12 & 8 & 6 & 18 & 11 & 10 & 4 & 8 \\
\hline 6 & 0 & 0 & 1 & 16 & 7 & 18 & 13 & 19 & 4 & 11 & 0 & 3 & 18 \\
\hline 7 & 0 & 0 & 1 & 7 & 14 & 15 & 6 & 13 & 2 & 15 & 7 & 10 & 10 \\
\hline 8 & 0 & 0 & 3 & 12 & 6 & 7 & 14 & 7 & 8 & 15 & 18 & 4 & 6 \\
\hline 9 & 0 & 1 & 2 & 7 & 8 & 8 & 5 & 11 & 28 & 8 & 11 & 3 & 8 \\
\hline 10 & 0 & 3 & 3 & 12 & 5 & 5 & 11 & 8 & 25 & 10 & 7 & 3 & 9 \\
\hline 11 & 0 & 0 & 0 & 6 & 1 & 3 & 0 & 14 & 16 & 0 & 56 & 1 & 3 \\
\hline 12 & 0 & 0 & 2 & 0 & 7 & 9 & 9 & 0 & 48 & 0 & 3 & 12 & 10 \\
\hline
\end{tabular}

Table 4

Identification rate (in \%) between the classes of the ground truth (first column) and the classes issued from the modelling of the two first-order moments (first line)

\begin{tabular}{|c|c|c|c|c|c|c|c|c|c|c|c|c|c|}
\hline & I & II & III & IV & $\mathrm{V}$ & VI & VII & VIII & IX & $\mathrm{X}$ & XI & XII & Other \\
\hline 1 & 81 & 0 & 0 & 6 & 0 & 4 & 0 & 0 & 0 & 0 & 0 & 0 & 9 \\
\hline 2 & 64 & 0 & 10 & 10 & 0 & 4 & 2 & 0 & 1 & 0 & 0 & 0 & 8 \\
\hline 3 & 29 & 0 & 27 & 7 & 0 & 15 & 4 & 1 & 1 & 0 & 0 & 0 & 15 \\
\hline 4 & 28 & 0 & 21 & 16 & 0 & 9 & 6 & 0 & 6 & 0 & 0 & 0 & 4 \\
\hline 5 & 12 & 0 & 24 & 27 & 0 & 13 & 4 & 1 & 5 & 2 & 0 & 0 & 12 \\
\hline 6 & 2 & 0 & 2 & 10 & 0 & 38 & 22 & 0 & 13 & 6 & 0 & 0 & 7 \\
\hline 8 & 8 & 0 & 1 & 19 & 1 & 7 & 13 & 1 & 16 & 15 & 0 & 13 & 6 \\
\hline 9 & 3 & 0 & 2 & 5 & 1 & 8 & 12 & 0 & 11 & 48 & 0 & 2 & 8 \\
\hline 10 & 3 & 0 & 2 & 2 & 1 & 4 & 11 & 0 & 17 & 42 & 0 & 7 & 11 \\
\hline 11 & 0 & 0 & 0 & 3 & 0 & 1 & 8 & 0 & 60 & 26 & 0 & 0 & 2 \\
\hline 12 & 0 & 0 & 0 & 7 & 0 & 2 & 1 & 0 & 25 & 57 & 0 & 0 & 8 \\
\hline
\end{tabular}




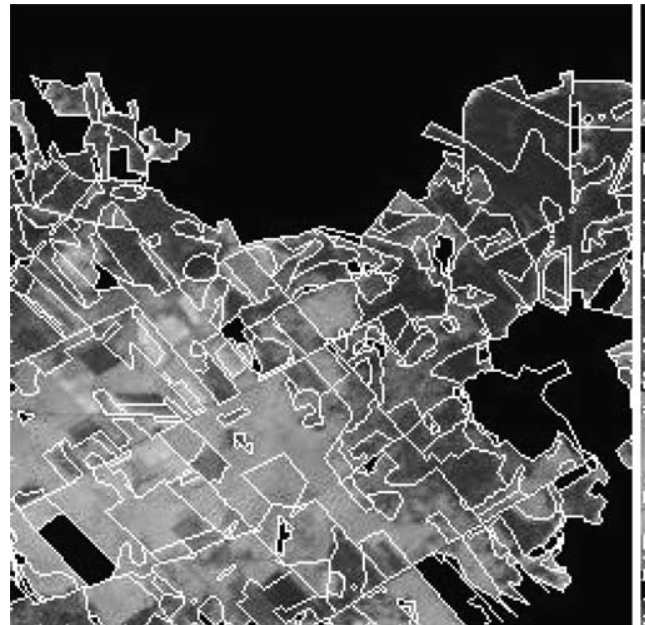

(a)

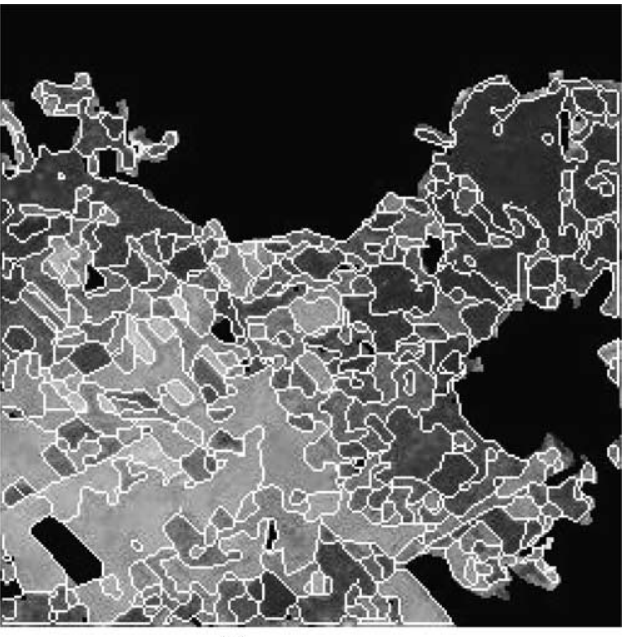

(b)

Fig. 10. Paimpont forest: (a) Ground truth. (b) Segmentation result. (Superimposed on the original image.)

associated statistics (means and variances in Table 1). Identical remarks can be made for the classes issued from the modelling of the two first-order moments (Table 4).

By considering that an identification rate greater than $40 \%$ is acceptable, we state that the modelling through the grey levels co-occurrence attributes and that through the two first-order moments yield better results: the most important classes (having more than 2500 pixels (Table 1) and corresponding to the classes 2, 5, 8, 9 and 10 of the ground truth (Fig. 11(a))) have been identified with relatively good rates.

On the whole, these results show that even though certain classes have been correctly identified, they still present weak percentages of classification. This is due in part, to the important shift between the ground truth rectilinear plots and the borders of certain regions in the original image (Fig. 10(a)).

\section{Conclusion}

In this paper, we have addressed the problem of detecting low-level features in images. In an iterative way, the suggested co-operative system allows us to obtain a segmentation in region and edge primitives. Following the classification of the different methods contained in the literature, the co-operation that we propose can be situated in the category of hybrid methods, related to the solution of artificial vision problems by using some concepts of 'data integration' and 'active perception'. It distinguishes itself from other approaches by the introduction of a mechanism of correction and adjustment by the bias of feedback and through the consideration of a measure of coherence of intermediate segmentation results. The system's objective is not explicitly defined for a specific application. The cooperation between the principle components (edge detection and region extraction) is effectuated in a bi-directional manner. It is realised from over-segmentations and evolves in the same direction until convergence towards compatible and coherent results occurs. Both the components produce appropriate results and that are improved through mutual information exchange. At the end of each cycle, the estimation of the coherence of the intermediate results is carried out with the aid of a dissimilarity measure, which considers both the detection and the positioning of the edges and the regions' boundaries.

The developed approach operates without intervention of high-level knowledge. It is almost automatic. It has been applied to both weakly and strongly textured real images, and the obtained results are conclusive. In the majority of examined cases, the efficiency of our approach expresses itself through a coherent detection of representative elements of the image. It has been particularly noted through good experimental results obtained on visible remote sensing imagery. Therefore, this approach confirms and shows the importance of:

- the complimentary primitives integration, which imply an abundance of information,

- the co-operation and the interaction in two directions,

- the adoption of a progressive working method in the formation of the primitives,

- the validation of each primitive aided by other primitives and the measure of its coherence, which leads to the extraction of accurate and pertinent primitives,

- and finally, the iterative parameter tuning, which allows their adaptation to the specificities of the analysed image.

In conclusion, the obtained results demonstrate the potential of the iterative co-operation scheme. Much work remains to be done in order to fully exploit the advantages of complementary primitives co-operation. Future efforts must be carried out by the introduction of other methods, taking into account the textural aspects of the regions, as well as the geometrical and structural aspects of the extracted primitives at different iterations. 


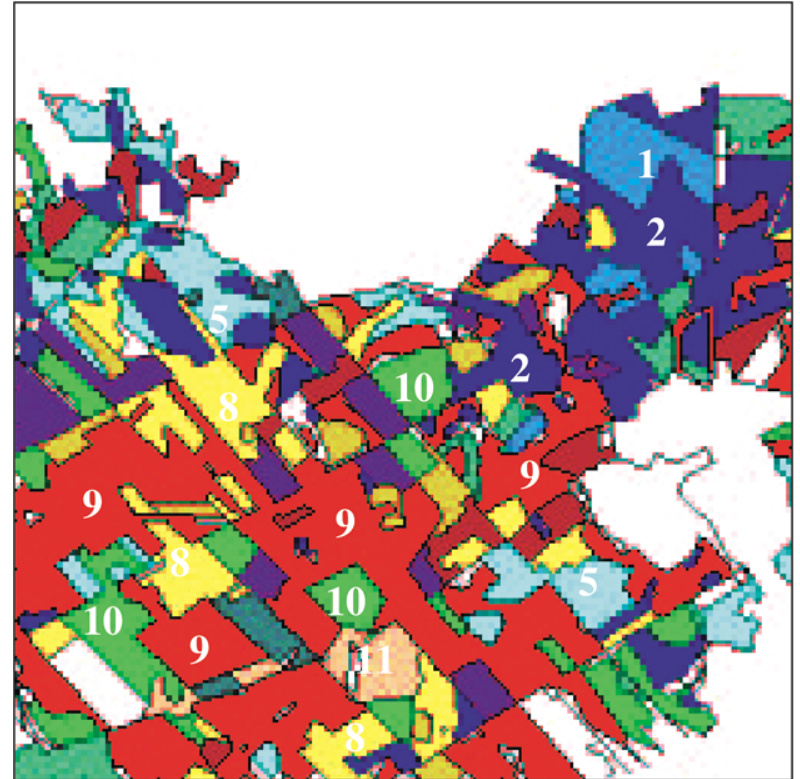

(a)

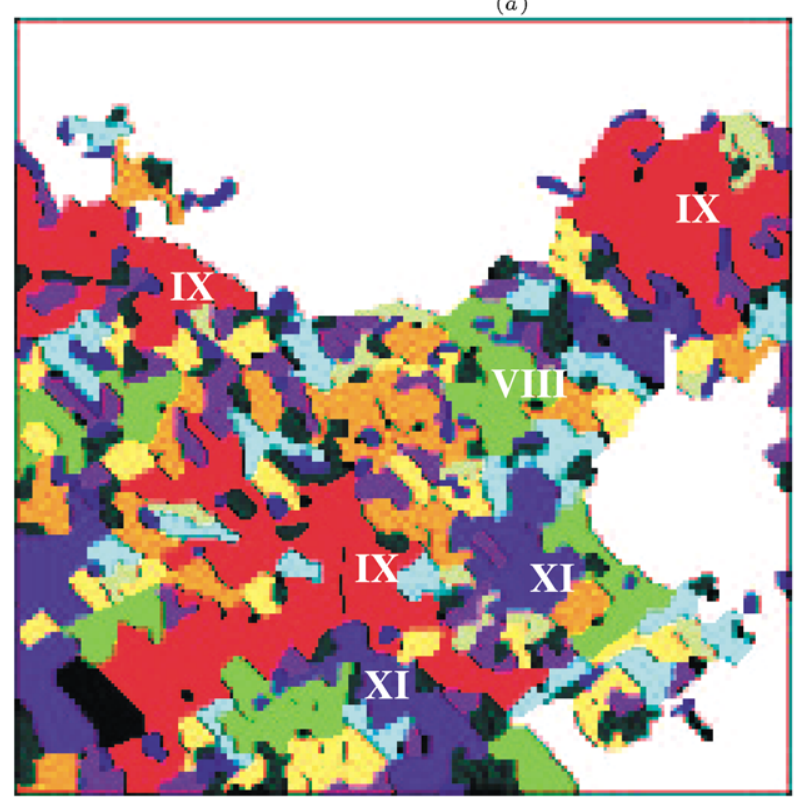

(c)

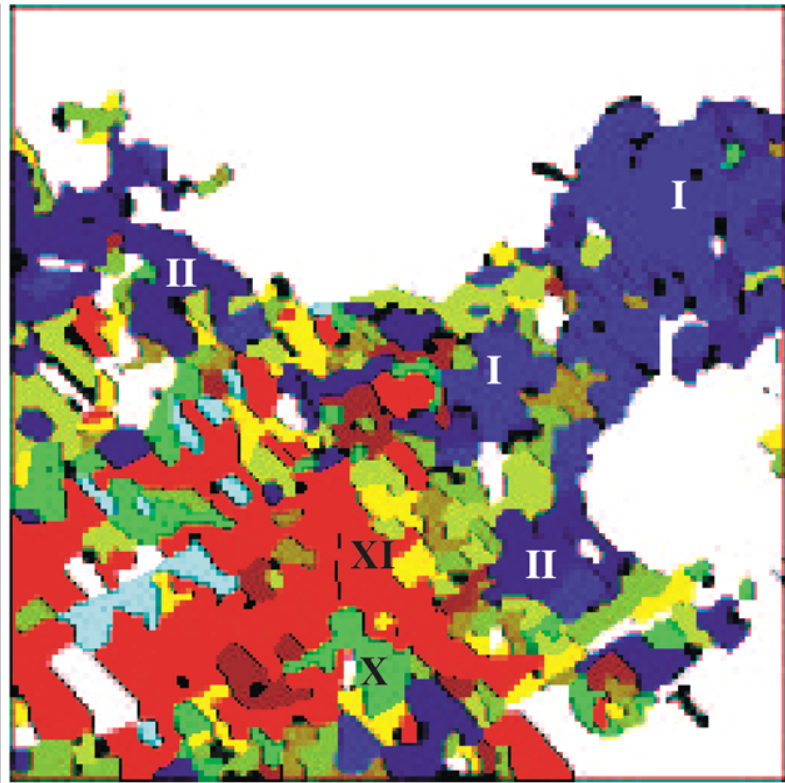

(b)

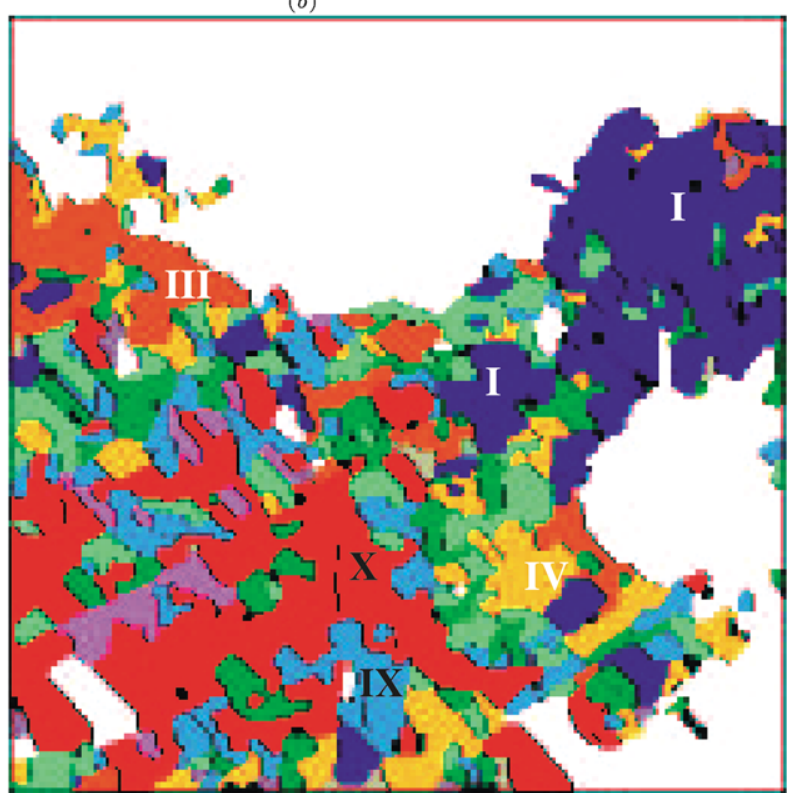

$(d)$

Fig. 11. Paimpont forest: (a) Ground truth map. Results after classification into 12 classes using (b) the co-occurrence attributes; (c) the run-length attributes; (d) the two first-order moments.

\section{References}

[1] N. Ahuja, A transform for multiscale image segmentation by integrated edge and region detection, IEEE Trans. Pattern Anal. Mach. Intell. 18 (12) (1996) 1211-1235.

[2] N. Ahuja, M. Tuceryan, Extractions of early perceptual structure in dot patterns: integrating region, boundary and component Gestalt, Pattern Recogn. 48 (1989) 304-356.

[3] A.J. Baddeley, An error metric for binary images, in: W. Forstner, S. Ruwiedel (Eds.), Proceedings of Robust Computer Vision, 1992, pp. $59-78$.

[4] R. Bajcsy, Active perception, Proc. IEEE 76 (8) (1988) 996-1005.

[5] J. Benois, D. Barba, Image segmentation by region-contour cooperation as basis for efficient coding scheme, Proceedings of the 11th ICPR, The Hague Nd, September, 1992, pp. 331-334.
[6] P. Bonnin, B. Zavidovique, La segmentation coopérative: comment combiner détection de contours et croissance de régions? Proceedings of 14th GRETSI Symposium Juan-les-Pins, September, 1993, pp. $755-758$.

[7] J. Canny, A computational approach to edge detection, IEEE Trans. Pattern Anal. Mach. Intell. 8 (6) (1986) 679-698.

[8] J.M. Chassery, Y. Elomary, Coopération contours actifs et multirésolution en segmentation d'images, Proceedings of 15th GRETSI Symposium, Juan-les-Pins, September, 1995, pp. 593-596.

[9] K. Chehdi, Q.M. Liao, Détection de contours basé sur la perception visuelle en vue de la segmentation d'images, Proceedings of 14th GRETSI Symposium, Juan-les-Pins, September, 1993, pp. 793-742.

[10] C.C. Chu, J.K. Aggarwal, The integration of image segmentation maps using region and edge information, IEEE Trans. Pattern Anal. Mach. Intell. 15 (12) (1993) 1241-1255. 
[11] J.P. Cocquerez, S. Philipp, Analyse d'images: filtrage et segmentation, Collection Enseignement de la physique, Masson, Paris, 1995.

[12] M.G. Cox, J.M. Rehg, S. Hingorani, A Bayesian multiple-hypothesis approach to edge grouping and contour segmentation, Int. J. Comput. Vision 11 (1) (1993) 5-24.

[13] R. Deriche, Fast algorithms for low-level vision, IEEE Trans. Pattern Anal. Mach. Intell. 18 (1) (1990) 679-683.

[14] P. Gamba, R. Lodola, A. Mecocci, Scene interpretation by fusion of segment and region information, Image Vision Comput. 15 (7) (1997) 499-509.

[15] M. Gokmen, C.C. Li, Edge detection and surface reconstruction using refined regularization, IEEE Trans. Pattern Anal. Mach. Intell. 15 (5) (1993) 492-499.

[16] J.P. Gombotto, A new approach to combining region growing and edge detection, Pattern Recogn. Lett. 14 (1993) 869-875.

[17] R.C. Gonzalez, P. Wintz, Digital Image Processing, second ed., Addison-Wesley, Reading, MA, 1992.

[18] J.F. Haddon, J.F. Boyce, Image segmentation unifying region and boundary information, IEEE Trans. Pattern Anal. Mach. Intell. 12 (10) (1990) 929-948.

[19] R.M. Haralick, L.G. Shapiro, Survey, image segmentation techniques, Computer Vision, Graphics and Image Processing, vol. 29, 1985, pp. $100-132$.

[20] D.P. Huttenloocher, G. Klanderman, J. Rucklidge, Comparating images using the Hausdorff distance, IEEE Trans. Pattern Anal. Mach. Intell. 15 (9) (1993) 850-863.

[21] R. Kara-Falah, P. Bolon, J.P. Cocquerez, A region-region and region-edge cooperative approach of image segmentation, Proc. ICIP 3 (1994) 470-474

[22] C.D. Kermad, Segmentation d'images: recherche d'une mise en œuvre automatique par coopération de méthodes, PhD Thesis, University of Rennes I, 1997.

[23] C.D. Kermad, K. Chehdi, C. Cariou, Image segmentation by an iterative region-contour control minimising a convergence criterion, Proceedings of ICSP, Bejing, October, 1996, pp. 131-134.

[24] I.Y. Kim, H.S. Yang, An integration scheme for image segmentation and labeling based on Markov random field model, IEEE Trans. Pattern Anal. Mach. Intell. 18 (1) (1996) 69-73.

[25] V. Koivunen, M. Pietikainen, Combined edge and region based method for range image segmentation, Proceedings of SPIE International Robotics Computer Vision Conference, Boston, November, vol. IX, 1990, pp. 501-512.

[26] J. Lemoigne, J.C. Tilton, Refining image segmentation by integration of edge and region data, IEEE Trans. Geosci. Remote Sensing 33 (3) (1995) 605-615.

[27] Q.M. Liao, Detection de contours et segmentation d'images, application à la télédétection et à la biologie marine, $\mathrm{PhD}$ Thesis, University of Rennes I, 1995.

[28] D.G. Lowe, Perceptual Organization and Visual Recognition, Kluwer Academic Publishers, Hingham, MA, 1985.

[29] W.Y. Ma, B.S. Manjunath, Edge flow: a framework of boundary detection and image segmentation, Proceedings of CVPR'97, 1997, pp. 744-749.

[30] D. Marr, E. Hildreth, Theory of edge detection, Proc. R. Soc. LoNd, Ser. B 207 (1980) 187-217.
[31] J.L. Marroquin, S. Mitter, T. Poggio, Probalistic solution of three illposed problems in computational vision, J. Am. Stat. Assoc. 82 (397) (1987) 76-89.

[32] I. Matalas, R. Benjamin, R. Kitney, An edge detection technique using the facet model and parameterized relaxation labeling, IEEE Trans. Pattern Anal. Mach. Intell. 19 (4) (1997) 328-341.

[33] M. Melkemi, J.M. Chassery, Edge-region segmentation processing based on generalized voronoï diagram representation, Proceedings of 11th ICPR, The Hague Nd, September, 1992, pp. 335-339.

[34] A. Mitiche, J.K. Aggarwal, Image segmentation by conventional and information integration techniques, Image Vision Comput. 3 (2) (1985) 50-60.

[35] V.S. Nalwa, E. Pauchon, Edge aggregation and edge description, Computer Vision, Graphics and Image Processing, vol. 40, 1987, pp. $79-94$.

[36] A. Nazif, M.D. Levine, Low-level segmentation: an expert system, IEEE Trans. Pattern Anal. Mach. Intell. 7 (2) (1985) 555-577.

[37] N.R. Pal, S.K. Pal, A review on image segmentation techniques, Pattern Recogn. 26 (1993) 1277-1294.

[38] T. Pavlidis, Y.T. Liow, Integration region growing and edge detection, IEEE Trans. Pattern Anal. Mach. Intell. 12 (3) (1990) 225-233.

[39] T.R. Reed, J.M.H. Du Buf, A review of recent texture segmentation and feature extraction techniques, Computer Vision, Graphics and Image Processing: Image Understanding, vol. 57, 1993, pp. 359-372.

[40] Revue Photo-Interpretation No. 1993/4-1994/1-2, vol. 32, ESKA, 1993.

[41] P.K. Sahoo, S. Soltani, A.K.C. Wong, Y.C. Chen, A survey of thresholding techniques, Computer Vision, Graphics and Image Processing, vol. 41, 1988, pp. 233-260.

[42] C. Spinu, C. Garbay, J.M. Chassery, Une approche coopérative et adaptative pour la segmentation d'images, Proceedings of 15th GRETSI Symposium, Juan-les-Pins, September, 1995, pp. 609-612.

[43] M. Tabb, N. Ahuja, Multiscale image segmentation by integrated edge and region detection, IEEE Trans. Image Process. 6 (5) (1997) $642-655$.

[44] A.N. Tikhonov, V.Y. Arsenin, Méthodes de Résolution de Problèmes mal Posés, MIR, Moscow, 1974.

[45] V. Torre, T. Poggio, On edge detection, IEEE Trans. Pattern Anal. Mach. Intell. 8 (1986) 679-698.

[46] B. Wrobel, O. Monga, Segmentation d'images naturelles: coopération entre un détecteur de contour et un détecteur de région, Proceedings of COGNITIVA MARI Conference, Paris, May, 1987, pp. 181-188.

[47] Y. Xiaohan, J. Yla-Jaaski, Image segmentation combining region growing and edge detection, Proceedings of 11th ICPR, The Hague Nd, September, 1992, pp. 339-342.

[48] P. Zamperoni, Analysis of region growing operators for image segmentation, in: Cappellini, et al. (Eds.), Advances in Image Processing and Pattern Recognition, Elseiver, Amsterdam, 1986.

[49] S.C. Zhu, A. Yuille, Region competition: unifying snakes, region growing and bayes/MDL for multiband image segmentation, IEEE Trans. Pattern Anal. Mach. Intell. 18 (9) (1996) 884-900.

[50] D. Ziou, S. Tabbone, Edge detection techniques-an overview, Int. J. Pattern Recogn. Image Anal. 8 (4) (1998) 537-559. 